\title{
Acute, isolated fractures of the metatarsal bones: an epidemiologic study
}

\author{
Viktoria Herterich $^{1} \cdot$ Luzie Hofmann $^{1} \cdot$ Wolfgang Böcker $^{1} \cdot$ Hans Polzer $^{1}\left(\mathbb{D} \cdot\right.$ Sebastian Felix Baumbach $^{1}$
}

Received: 17 December 2021 / Accepted: 14 February 2022 / Published online: 2 March 2022

(c) The Author(s) 2022, corrected publication 2023

\begin{abstract}
Introduction Although metatarsal fractures are common, the significance of previous epidemiologic studies is limited to specific fracture entities, subpopulations, or heterogeneous fracture aetiologies. The aim of the study was to assess the epidemiology of isolated metatarsal fractures in an adult population at a level-1 trauma centre.

Materials and methods Radiological and clinical databases were searched for a five-year period. Eligible were all patients with acute isolated metatarsal fractures over the age of 18 years with radiographs in two planes available. Stress fractures, injuries affecting Lisfranc joint stability, and concomitant injuries to other regions than the metatarsals were excluded. Data collection included general demographics, mechanism of injury, season of the trauma and fracture details.

Results Out of 3259 patients, 642 patients met the inclusion criteria and were included for the analysis. The patients' mean age was $44.5 \pm 18.9$ years, $50.6 \%$ were female. $83.3 \%$ suffered an isolated, $16.7 \%$ multiple metatarsal fractures. Single metatarsal fractures occurred predominantly at the fifth metatarsal bone (81.3\%), their frequency decreased with increasing age, with a seasonal peak during the summer. Patients suffering multiple metatarsal fractures were significantly older $(51.6 \pm 21.2$ vs. $43.0 \pm 18.1$ years; $p<0.001)$ and the injury resulted significantly more often from a high-energy trauma ( $6.7 \%$ vs. $23.4 \%$; $p<0.001)$. Multiple metatarsal fractures occurred evenly throughout all metatarsals but revealed a focus on female population with no seasonal differences.

Conclusion Single metatarsal fractures predominantly occurred at the fifth metatarsal bone and showed a seasonal, gender and age dependency. Multiple metatarsal fractures were homogeneously distributed between the different metatarsals with distinct age-dependent gender differences.
\end{abstract}

Level of evidence Level III.

Keywords Metatarsal $\cdot$ Fracture $\cdot$ Epidemiology $\cdot$ Fracture distribution

\section{Introduction}

Metatarsal fractures belong to the ten most common fractures with a prevalence of 3.2-6.8\% of all fractures with an annual incidence of 67-75.4/100.000 per year [3, 7, 17, 21]. Furthermore, they account for up to $88.5 \%$ of all fractures to the foot $[8,22,23]$. Despite their frequency, epidemiological data on metatarsal fractures are rare, as most recent literature focusses on treatment recommendations, especially for the

Hans Polzer

hans.polzer@med.uni-muenchen.de

1 Department of Orthopaedics and Trauma Surgery, Musculoskeletal University Center Munich (MUM), University Hospital, LMU Munich, Ziemssenstr. 5, 80336 Munich, Germany base of the fifth metatarsal and lacks epidemiological data $[1,9,11,15]$.

The significance of previous epidemiological studies on metatarsal fractures is limited as they either focused on specific entities (fractures to the base of the fifth metatarsal or dancer's fractures), evaluated subpopulations only (elderly Caucasian women or children), or included heterogeneous fracture aetiologies (including concomitant injuries/stress fractures) $[2,4,5,8,13,16,17,19,21]$. Consequently, we are still missing valid epidemiological data of a well-characterized cohort for one of the most common fractures in adulthood.

Therefore, the aim of this study was to assess the epidemiology of isolated metatarsal fractures in an adult population over a 5-year period at a level-1 trauma centre. 


\section{Materials and methods}

The herein presented work is a retrospectively radiographicepidemiological study. The study was approved by the local ethics committee (\#20.0442).

\section{Patient screening}

The hospital's clinical database was searched per the ICD10 S92.0 code and the radiological database per the search terms: ("metatars*" OR "midfoot*") AND ("fracture" OR "bon* injury") between 01.01.2015 and 12.31.2019. Inclusion criteria were adult patients ( $\geq 18$ years) with acute isolated metatarsal fracture(s) and plain radiographs of the foot in two planes available within the first 14 days of the injury. Exclusion criteria were stress fractures, defined as slight fractures without relevant trauma, injuries affecting Lisfranc joint stability, and any concomitant injury to other regions than the metatarsals.

\section{Patient selection}

The results of the clinical and radiological database were merged, and duplicates were removed. The removal of the duplicates was performed after merging the results; therefore, it remains unclear if the duplicates were within each database or between both databases. The resulting database was screened for eligible patients, independently by two blinded investigators ( $\mathrm{VH}, \mathrm{LH})$. In case of disagreement, the conflict was resolved by discussion with the senior author (SFB).

\section{Data collection}

From all eligible patients, the following data points were collected: general demographics, mechanism of injury (high energy/low energy) - high energy was defined as every trauma mechanism beyond supination force on plane ground-season of accident, and fracture details including side fracture, number of metatarsals fractured, and fracture location (proximal, shaft, distal).

\section{Statistics}

Next to general demographics, group comparisons were conducted using Pearson Chi-Square, independent sample t-test, oneway ANOVA with Tukey post hoc analysis were appropriate. Due to multiple testing, a Bonferroni correction was conducted setting the level of significance to $p<0.01$. Data are presented as mean \pm standard deviation, if not stated differently. All statistics were computed using SPSS (26.0, IBM).

\section{Results}

In the radiographic database, 3716 patients were identified and in the clinical database, 936 patients were identified. Removal of duplicates $(n=1393)$ resulted in 3259 patients. Following independent, blinded review, 642 patients were eligible for final analysis according to the inclusion criteria (Fig. 1).

The patients' mean age was $44.5 \pm 18.9$ years, $50.6 \%$ were female, and the left side was fractured in $50.2 \%$ of patients. An overview of the general demographics and fracture distribution is provided in Table 1. 535 patients (83.3\%) suffered an isolated metatarsal fracture. In $16.7 \%$ of the patients, more than one metatarsal bone was fractured. Patients with multiple metatarsal fractures were significantly older $(51.6 \pm 21.2$ vs. $43.0 \pm 18.1$ years; $p<0.001)$ and the injury resulted significantly more often from a high-energy accident $(6.7 \%$ vs. $23.4 \% ; p<0.001)$ compared to patients with a single metatarsal fracture. No significant differences were found for sex $(p=0.459)$ or the fractured side $(p=1)$.

Out of all fractures, $4.1 \%$ occurred at the first metatarsal (MT), $10.3 \%$ at MT II, $11.8 \%$ at MT III, $14.3 \%$ at MT IV, and $59.6 \%$ at MT V. The fracture distribution (proximal, shaft, distal) per the number of the metatarsals fractured (single/multiple) differed significantly $(p<0.001)$ and is outline in Fig. 2. Whereas multiple metatarsal fractures showed a rather homogeneous distribution, single metatarsal fractures occurred predominantly $(81.3 \%)$ within the fifth metatarsal bone.

Out of all isolated fifth metatarsal fractures, $72.5 \%$ occurred proximal, $20.8 \%$ in the shaft region and $6.7 \%$ distally. Fracture locations within the fifth metatarsal were independent of age $(p=0.083)$, sex $(p=0.171)$, fractured side $(p=0.355)$, or trauma mechanism $(p=0.012)$. Regarding the proximal fractures to the fifth metatarsal, the distribution according to the Lawrence and Botte classification was: Type I: $63.0 \%$, Type II: $21.8 \%$, Type III: $15.2 \%$ [12].

Next, the age distribution was analysed. Overall, a moderate negative correlation was found between age and the number of patients $(r=-0.581 ; p<0.001)$ or the number of metatarsal fractures $(r=-0.712 ; p<0.001)$. This moderate negative correlation stayed true when conducting the gender specific analysis (Fig. 3A). Interestingly, there was a higher degree of correlation for male compared to female patients, both when analysing the number of patients and the number of metatarsal fractures.

Then, the dataset was analysed separately for single or multiple metatarsal fractures and their correlation to age. For single metatarsal fractures, an overall high correlation was found $(r=-0.951 ; p<0.001)$, which stayed true when analysed separately for gender (Fig. 3B). For multiple metatarsal fractures, a moderate negative correlation was 


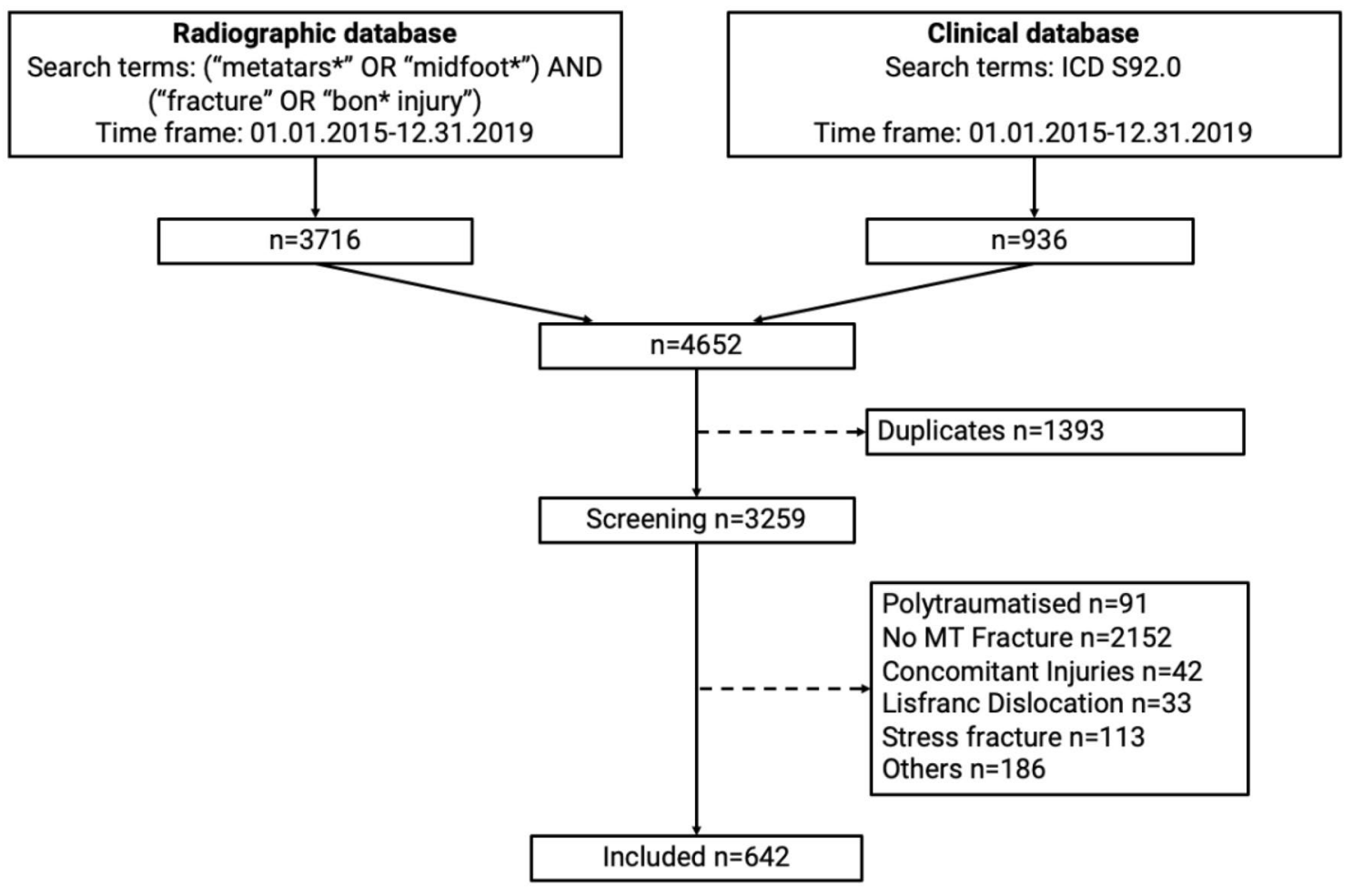

Fig. 1 Patient selection flow chart. $n$ number, $M T$ metatarsal. Other reasons: delayed presentation; missing initial X-ray; date of Injury; $>2019 /<2015$

Table 1 General demographic overview of different fracture combination and distributions

\begin{tabular}{|c|c|c|c|c|c|c|}
\hline $\begin{array}{l}\text { Number of metatar- } \\
\text { sals fractured }\end{array}$ & $\begin{array}{l}\text { Number of patients } \\
\text { [percentage }{ }^{\mathrm{a}} \text { ] }\end{array}$ & $\begin{array}{l}\text { Most common combination } \\
\left.\text { [percentage }^{b}\right]\end{array}$ & Age $[$ mean $\pm S D]$ & Sex [\% female] & Side $[\%$ left $]$ & $\begin{array}{l}\text { Percent } \\
\text { high } \\
\text { energy }\end{array}$ \\
\hline 1 & 535 & - & $43.0 \pm 18.1$ & $49.9 \%$ & $50.1 \%$ & 6.7 \\
\hline 2 & $61[57.0 \%]$ & $\mathrm{IV}+\mathrm{V}[45.9 \%]$ & $54.7 \pm 21.7$ & $62.3 \%$ & $45.9 \%$ & 14.8 \\
\hline 3 & $32[29.9 \%]$ & $\mathrm{II}+\mathrm{III}+\mathrm{IV}[58.3 \%]$ & $42.6 \pm 18.8$ & $43.8 \%$ & $46.9 \%$ & 28.1 \\
\hline 4 & $13[12.1 \%]$ & $\mathrm{II}+\mathrm{III}+\mathrm{IV}+\mathrm{V}[68.8 \%]$ & $57.2 \pm 22.7$ & $38.5 \%$ & $76.9 \%$ & 46.2 \\
\hline 5 & $1[0.9 \%]$ & - & [77] & {$[100 \%]$} & {$[100 \%]$} & [100\%] \\
\hline
\end{tabular}

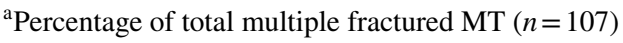

${ }^{\mathrm{b}}$ Percentage within that group

found for age and both, the number of patients $(r=-0.526$; $p=0.036)$ as well as the number of fractured metatarsals $(r=-0.544 ; p=0.029)$. Interestingly, again a high negative correlation was found for male patients and the number of patients and the number of fractures (Fig. 3C). No correlation was observed for female patients and for the number of patients or fractures. Observationally, female patients rather showed two age peaks, one between 40 and 49 years and the other between 70 and 79 years.

Finally, the season distribution for metatarsal fractures was investigated. Looking at all metatarsal fractures (Fig. 3D), there appears to be a gender-independent peak during summer and a low during winter. A similar trend was found for single metatarsal fractures (Fig. 3E). When looking at multiple metatarsal fractures, a more homogeneous distribution was observed with trending lower numbers during fall and winter when compared to spring and summer (Fig. 3F).

\section{Discussion}

This study is the first to investigate the epidemiology of isolated metatarsal fractures in a large adult population. Significant differences were found for the anatomical location, 




Fig. 2 Distribution per the number of metatarsals fractures. MT metatarsal
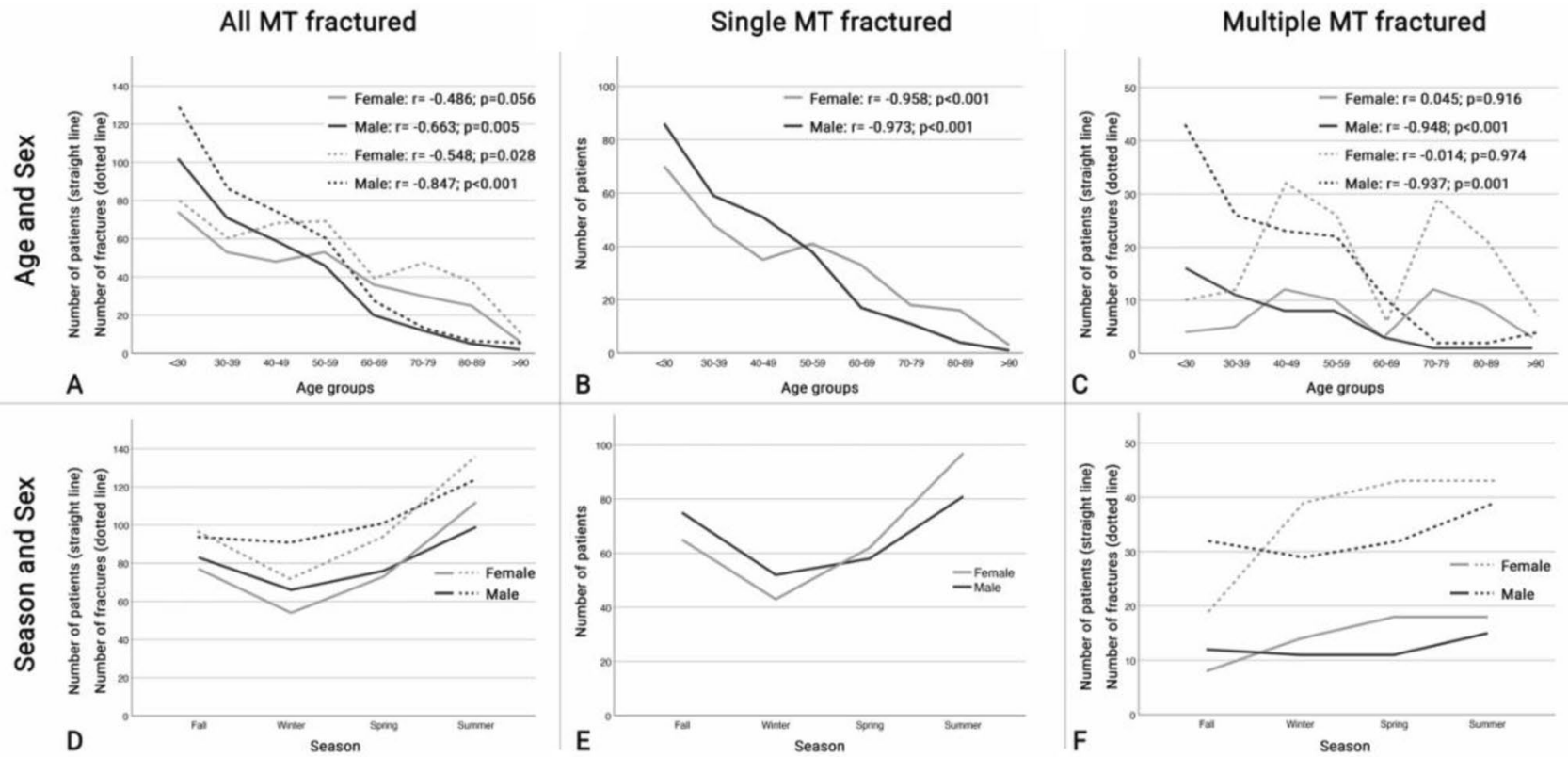

Fig. 3 Gender-specific age and season distribution of metatarsal fractures. A Age- and sex-specific distribution of single metatarsal fractures; B Age- and sex-specific distribution of multiple metatarsal

age, and seasonal distribution between single and multiple fractured metatarsals. Single metatarsal fractures showed a distinct age-dependent decrease and a peak during summer. Multiple metatarsal fractures revealed a sex dependent age distribution, with fractures in females occurring predominantly between $40-49$ and $70-79$ years. fractures; C Season- and sex-specific distribution of single metatarsal fractures; D Season- and sex-specific distribution of multiple metatarsal fractures

The presented dataset of 624 adult patients suffering an isolated metatarsal fracture compares favorably to literature. Previous epidemiologic studies comprised around 400 patients and furthermore presented heterogeneous patient populations [2, 17]. An exception could be the study by Zhao et al. [24] who apparently included 1949 adult metatarsal 
fractures. Unfortunately, the study was published in Chinese and could therefore not be reviewed.

The overall distribution of metatarsal fractures reported herein, i.e., a rather homogenous distribution between MT I and IV fractures and a clear peak in MT V fractures has been stated before $[2,17,18]$. When analyzing the whole sample, $59.6 \%$ of metatarsal fractures were located at the fifth metatarsal, which is well in line with data published previously, varying between 43 and 68\% [6, 10, 17, 18, 20, 24]. Interestingly, when subdividing the whole population between multiple and single metatarsal fractures, only $17.7 \%$ of all multiple but $81.3 \%$ of single metatarsal fractures occurred at the fifth metatarsal.

Furthermore, also the fracture location distribution (proximal-shaft-distal) within the fifth metatarsal bone varied between single- and multiple-metatarsal fractures. Previous studies have reported a dominance of proximal metatarsal fractures, ranging from 49.8 to $72 \%$ of all MT V fractures [6, $10]$. When conducting this analysis separately for single and multiple metatarsal fractures, we found a rather homogeneous distribution for multiple MT fractured but a predominance of proximal MT V fractures for isolated MT fractures.

The authors are aware of two studies only, which discriminated single and multiple metatarsal fractures $[2,17]$. They reported that $7.8-15.6 \%$ of all metatarsal fractures affected multiple metatarsals. However, these studies did not perform further analyses. Due to the herein observed differences between single and multiple metatarsal fractures, future epidemiological studies should incorporate not only a plane description of fracture frequency but also possible differences between different fracture etiologies and patterns.

Next, the influence of the patients' age on the occurrence of metatarsal fractures was analyzed. Previous studies have reported an age-dependent decrease in metatarsal fractures $[2,3,10,17,20]$. Moreover, they have stated an additional age- and gender-dependent distribution. For male patients, metatarsal fractures were reported to peak in their thirties. For female patients' metatarsal fractures were found to peak between the age 50 and 70 years $[2,10,14,18]$. One major limitation of these studies was again, that they did not discriminate between single and multiple metatarsal fractures. In the herein published cohort, a comparable trend was observed when analyzing the whole sample (Fig. 3A). Fractures in male patients showed a constant decrease in patients aged 30 years and above. Fractures in female patients showed two peaks, at the ages of 50-59 years and 70-79 years. When differentiating single and multiple metatarsal fractures, one does see a different, but more congruent picture. In single metatarsal fractures, a high negative correlation was found between age and both, male $(r=-0.973$; $p<0.001)$ and female $(r=-0.958 ; p<0.001)$ patients (Fig. 3B). For multiple metatarsal fractures, again a high negative correlation was found between age and number of patients $(r=-0.948 ; p<0.001) /$ of metatarsal fractures $(r=-0.937 ; p=0.001)$ in male patients. But no correlation between age and female number of patients $(r=0.045$; $p=0.916) /$ metatarsal fractures $(r=-0.014 ; p=0.974)$, but rather two clear peaks between $40-49$ years and $70-79$ years. This age and gender-dependent distribution has considerable implications for the clinical routine as well as future studies. Whereas single metatarsal fractures apparently show an agedependent decrease, multiple metatarsal fractures in female show peaks similar to those in osteoporosis-associated fractures. Therefore, one can hypothesis, that female patients suffering multiple metatarsal fractures might be at higher risk for osteoporosis.

When investigating the season-dependent fracture distribution, the differentiation between single and multiple metatarsal fractures again proofed important. Whereas the cumulative (Fig. 3D) and single metatarsal fracture (Fig. 3) analysis showed a peak for the summer and low for the winter, a considerably more homogeneous distribution was found for multiple metatarsal fractures. This again raises the suspicion, that pathomechanisms of isolated multiple metatarsal fractures might be different to those of single metatarsal fractures. Explanation could be the above mentioned, age dependent, alteration in bone metabolism, i.e., osteoporosis or the higher number of high energy accidents. Further studies are needed to proof the hypothesis that metatarsal fractures could be osteoporosis-associated fractures.

\section{Limitations of the study}

The major limitation of this study is its descriptive design. Still, for the first time, we were able to provide a comprehensive picture of the epidemiology of isolated metatarsal fractures in an adult population.

Furthermore, the study has no direct clinical implementation. But researchers face a similar problem when conducting descriptive or retrospective studies. Still, the detailed epidemiological analysis of metatarsal fractures does not only have an academic purpose but does have considerable implications for future studies. As outlined above, future studies should investigate a possible correlation between multiple isolated metatarsal fractures and osteoporosis. Considering their early age peak between 40 and 49 years, they might be identified as early indicator fractures for osteoporosis.

\section{Strengths of the study}

First to mention is this study's clearly defined patient population assessing only isolated metatarsal fractures in adult patients and excluding all concomitant ligamentous and bony injuries. Therefore, a more decisive statement can be made about the thus very determined fracture types. 
Furthermore, the review of patients was performed by two independent reviewers and conflicts were solved by consulting the senior author; therefore, a very strict and even patient selected was achieved.

Finally, a detailed analysis of the fracture patterns was performed. Looking at the results reported, it seems extremely important to differentiate between single and multiple MT fractures as they differed significantly.

\section{Conclusion}

This is the largest epidemiological studies on isolated metatarsal fractures in adult patients. Based on the data available, a differentiation between the two entities single and multiple isolated metatarsal fractures seems necessary. Whereas single metatarsal fractures predominantly occur at the fifth metatarsal bone and show a seasonal dependency, multiple metatarsal fractures revealed a rather homogeneous distribution between the different metatarsal bones with distinct ageand gender-dependent differences. Future studies should not only emphasis the differentiation between single and multiple isolated metatarsal fractures, but also investigate a possible correlation between multiple isolated metatarsal fractures in female patients and osteoporosis.

Funding Open Access funding enabled and organized by Projekt DEAL. The author(s) received no financial support for the research, authorship, and/or publication of this article.

\section{Declarations}

Conflict of interest The author(s) declared no potential conflicts of interest with respect to the research, authorship, and/or publication of this article. ICMJE forms for all authors are available online.

Ethical approval The study was approved by the local ethic committee. The study was performed at Department of Orthopaedics and Trauma Surgery, Musculoskeletal University Center Munich (MUM), University Hospital, LMU Munich.

Open Access This article is licensed under a Creative Commons Attribution 4.0 International License, which permits use, sharing, adaptation, distribution and reproduction in any medium or format, as long as you give appropriate credit to the original author(s) and the source, provide a link to the Creative Commons licence, and indicate if changes were made. The images or other third party material in this article are included in the article's Creative Commons licence, unless indicated otherwise in a credit line to the material. If material is not included in the article's Creative Commons licence and your intended use is not permitted by statutory regulation or exceeds the permitted use, you will need to obtain permission directly from the copyright holder. To view a copy of this licence, visit http://creativecommons.org/licenses/by/4.0/.

\section{References}

1. Bean BA, Smyth NA, Abbasi P, Parks BG, Hembree WC (2021) Biomechanical comparison of hook plate vs headless compression screw fixation of large fifth metatarsal base avulsion fractures. Foot Ankle Int 42(1):89-95

2. Cakir H, Van Vliet-Koppert S, Van Lieshout E, De Vries M, Van Der Elst M, Schepers T (2011) Demographics and outcome of metatarsal fractures. Arch Orthop Trauma Surg 131(2):241-245

3. Court-Brown CM, Caesar B (2006) Epidemiology of adult fractures: a review. Injury 37(8):691-697

4. Dameron TB Jr (1995) Fractures of the proximal fifth metatarsal: selecting the best treatment option. J Am Acad Orthop Surg 3(2):110-114

5. Egol K, Walsh M, Rosenblatt K, Capla E, Koval KJ (2007) Avulsion fractures of the fifth metatarsal base: a prospective outcome study. Foot Ankle Int 28(5):581-583

6. Ekrol I, Court-Brown C (2004) Fractures of the base of the 5th metatarsal. Foot 14(2):96-98

7. Emmett JE, Breck LW (1958) A review and analysis of 11,000 fractures seen in a private practice of orthopaedic surgery 1937 1956. JBJS 40(5):1169-1175

8. Hasselman CT, Vogt MT, Stone KL, Cauley JA, Conti SF (2003) Foot and ankle fractures in elderly white women: incidence and risk factors. JBJS 85(5):820-824

9. Herterich V, Baumbach SF, Kaiser A, Böcker W, Polzer H (2021) Fifth metatarsal fracture: a systematic review of the treatment of fractures of the base of the fifth metatarsal bones. Dtsch Arztebl Int 118(35-36):587

10. Kane JM, Sandrowski K, Saffel H, Albanese A, Raikin SM, Pedowitz DI (2015) The epidemiology of fifth metatarsal fracture. Foot Ankle Spec 8(5):354-359

11. Khurana A, Alexander B, Pitts C et al (2020) Predictors of malreduction in zone II and III Fifth metatarsal fractures fixed with an intramedullary screw. Foot Ankle Int 41(12):1537-1545

12. Lawrence SJ, Botte MJ (1993) Jones' fractures and related fractures of the proximal fifth metatarsal. Foot Ankle 14(6):358-365

13. Lehman RC, Torg JS, Pavlov H, DeLee JC (1987) Fractures of the base of the fifth metatarsal distal to the tuberosity: a review. Foot Ankle 7(4):245-252

14. Lievers WB, Goggins KA, Adamic P (2020) Epidemiology of foot injuries using national collegiate athletic association data from the 2009-2010 through 2014-2015 seasons. J Athl Train 55(2):181-187

15. Looney AM, Renehan JR, Dean DM et al (2020) Rate of delayed union with early weightbearing following intramedullary screw fixation of Jones fractures. Foot Ankle Int 41(11):1325-1334

16. Nunley JA (2001) Fractures of the base of the fifth metatarsal: the Jones fracture. Orthop Clin North Am 32(1):171-180

17. Petrisor BA, Ekrol I, Court-Brown C (2006) The epidemiology of metatarsal fractures. Foot Ankle Int 27(3):172-174

18. Rasmussen CG, Jørgensen SB, Larsen P, Horodyskyy M, Kjær IL, Elsoe R (2021) Population-based incidence and epidemiology of 5912 foot fractures. Foot Ankle Surg 27(2):181-185

19 Schwagten K, Gill J, Thorisdottir V (2020) Epidemiology of dancers fracture. Foot Ankle Surg. https://doi.org/10.1016/j.fas.2020. 09.001

20. Shuen W, Boulton C, Batt M, Moran C (2009) Metatarsal fractures and sports. Surg 7(2):86-88

21. Singer G, Cichocki M, Schalamon J, Eberl R, Höllwarth ME (2008) A study of metatarsal fractures in children. JBJS 90(4):772-776

22. Urteaga A, Lynch M (1995) Fractures of the central metatarsals. Clin Podiatr Med Surg 12(4):759-772 
23. Zenios M, Kim W, Sampath J, Muddu B (2005) Functional treatment of acute metatarsal fractures: a prospective randomised comparison of management in a cast versus elasticated support bandage. Injury 36(7):832-835

24. Zhao H, Wu H, Wu W et al (2010) Epidemiological investigation of adult metatarsal fractures. Zhonghua Yi Xue Za Zhi 90(1):15-18
Publisher's Note Springer Nature remains neutral with regard to jurisdictional claims in published maps and institutional affiliations. 